Leading opinion

\title{
Functionalized carbon nanotubes as immunomodulator systems
}

\author{
Mario Pescatori a, b , Davide Bedognetti ${ }^{c}$, Enrica Venturelli ${ }^{\mathrm{d}}$, Cécilia Ménard-Moyon ${ }^{\mathrm{d}}$, \\ Camilla Bernardini ${ }^{\mathrm{e}}$, Elena Muresu ${ }^{\mathrm{f}}$, Andrea Piana ${ }^{\mathrm{f}}$, Giorgio Maida ${ }^{\mathrm{f}}$, Roberto Manetti ${ }^{\mathrm{g}}$, \\ Francesco Sgarrella ${ }^{\mathrm{h}}$, Alberto Bianco ${ }^{\mathrm{d}, *}$, Lucia Gemma Delogu ${ }^{\mathrm{h}, *}$ \\ ${ }^{a}$ Department of Surgery, Section Surgical Oncology, Laboratory Experimental Surgical Oncology, Erasmus Medical Center, Rotterdam, The Netherlands \\ ${ }^{\mathrm{b}}$ Crosslinks BV, Rotterdam, The Netherlands \\ ${ }^{\mathrm{c}}$ Infectious Disease and Immunogenetics Section, Department of Transfusion Medicine, Clinical Center and Trans-National Institutes of Health Center for \\ Human Immunology, National Institutes of Health, Bethesda, MD 20892, USA \\ ${ }^{\mathrm{d}}$ Centre National de la Recherche Scientifique, Institut de Biologie Moléculaire et Cellulaire, Laboratoire d'Immunopathologie et Chimie Thérapeutique, \\ 67000 Strasbourg, France \\ e Institute of Anatomy and Cell Biology, Università Cattolica S. Cuore, Rome, Italy \\ ${ }_{\mathrm{f}}^{\mathrm{f}}$ Dipartimento di Scienze Biomediche, Università degli Studi di Sassari, 07100 Sassari, Italy \\ ${ }^{\mathrm{g}}$ Dipartimento di Medicina Clinica, Sperimentale e Oncologica, Università degli Studi di Sassari, 07100 Sassari, Italy \\ ${ }^{\mathrm{h}}$ Dipartimento di Chimica e Farmacia, Università degli Studi di Sassari, 07100 Sassari, Italy
}

\section{A R T I C L E I N F O}

\section{Article history:}

Received 6 February 2013

Accepted 19 February 2013

Available online 16 March 2013

\section{Keywords:}

Nanomedicine

Carbon nanomaterials

Immune system

Gene expression

Microarrays

Cytokines

\begin{abstract}
A B S T R A C T
In view of the broad potential biomedical applications of carbon nanotubes (CNTs) different studies were performed to assess their effect on the immune system. However, the work performed to date was able to give a restricted view looking only at some activation markers and cytokine expression. The immune system is rarely limited to few molecule interactions being instead always a balance of switching several genes on and off. Whole genome expression (microarray) is a technology able to give the full picture on genome expression. Here we describe a microarray genome-wide study on Jurkat cells, a T lymphocyte cell line, and THP1, a monocytic cell line, representative of both types of immune response, the adaptive and innate, respectively. Since any structure or molecule modification may lead to very different immune reactions, we treated the two cell lines with four types of functionalized multi-walled CNTs that differ in terms of functionalization and diameter. After having assessed the internalization and the lack of toxicity of CNTs in both cell types, we used the Affymetrix technology to analyze the expression of about 32,000 transcripts. Three of the tested nanotubes (i.e., ox-MWCNT-1, ox-MWCNT-NH ${ }_{3}^{+}-1$, and ox-MWCNT$\mathrm{NH}_{3}^{+}-2$ ) activated immune-related pathways in monocytes but not in $\mathrm{T}$ cells. In view of these charateristics they were named as monocyte activating CNTs (MA-CNTs). Molecular pathways upregulated by MA-CNTs included IL6, CD40, dendritic cell maturation, tumor necrosis factor-(TNF)- $\alpha /$ TNFR1-2, NFKB signaling and T helper 1 chemokine pathways (CXCR3 and CCR5 ligand pathways). These pathways are commonly activated during acute inflammatory processes as those associated with immune-mediated tumor rejection and pathogen clearance. One of them (i.e., ox-MWCNT-2) downregulated genes associated with ribosomal proteins in both monocytes and $\mathrm{T}$ cells. We validated our findings at gene expression level by performing real-time PCR assessing the most highly modulated genes in monocytes. To confirm the results at protein level, the secretion of IL1 $\beta$, TNF $\alpha$, IL6 and IL10 by THP1 and primary monocytes was assessed by ELISA, corroborating gene-expression data. Our results provide new insights into the whole gene expression modulation by different CNTs on immune cells. Considering the well known drug carrier ability of CNTs, our findings demonstrate that MA-CNTs here behave as cell specific immunostimulatory systems, giving very interesting future perspectives for their application also as immunotherapeutic agents and/or vaccine adjuvants.
\end{abstract}

(c) 2013 Elsevier Ltd. All rights reserved.

\footnotetext{
* Corresponding authors.

E-mail addresses: a.bianco@ibmc-cnrs.unistra.fr (A. Bianco), lgdelogu@uniss.it, luciadel82@yahoo.it (L.G. Delogu).
}

\section{Introduction}

In medicine, nanotechnology represents a promising strategy to identify new solutions to unsolved diseases and diagnostic problems. In the last few years, great expectations were placed into 
functionalized carbon nanotubes ( $f$-CNTs). Although nonfunctionalized (pristine) CNTs are highly cytotoxic, their functionalization allows enhancing solubility in water with consequent enhancement of their biocompatibility [1].f-CNTs were successfully investigated by us and others for several biomedical applications such as drug carriers [2-9], particularly for antisense oligonucleotide delivery against PTPN22 [10](a key gene for autoimmune disease [11]), as potential agent for kidney tumors treatment [12], and as scaffolds able to increase neuronal performance [13]. In addition, we recently described that functionalized CNTs could be potentially used as injectable ultrasound contrast agent [14]. Considering that therapeutic and diagnostic applications of CNTs would need a systemic administration, it is extremely important to be aware of their effect on peripheral immune cells, which are the first target encountered soon after their administration. A number of studies in the last few years have been aimed at understanding the possible impact of carbon nanotubes on the immune system [15-20]. Very little information is available about the global biological effect of on human immune cells [21]. In fact, studies performed so far were focused on few classic parameters of immune response such as activation markers and cytokines [15-22]. Even though these approaches can elucidate the effect of CNTs on specific immune molecules, they do not provide reliable insight into the global effect and could miss the full picture of CNT interactions. Similar considerations were also made on other nanomaterials [23]. The immune system is always a balance of switching genes on and off. The wholegenome gene expression profiling can detect modification of thousands of genes simultaneously. To our knowledge, no studies have investigated so far the effect of functionalized CNTs by this approach on human immune cells. Microarray technology was employed only by Chou et al. to study the effect of a single type of nonfunctionalized single-walled carbon nanotubes (SWCNTs) on THP1-derived macrophages showing substantial cytotoxicity [24].

In a previous study we screened the effect of a wide variety of $f$ CNTs on the major populations of immune cells by classical activation assays, showing that some of them were able to boost the immune response in human primary monocytes (i.e. enhancement of IL6 release and over-expression of CD25) [22]. Importantly, we did not detect any cytotoxic effect for all the tested $f$-CNTs. The aim of the present investigation was to assess whether different CNT functionalizations and diameters could determine distinct molecular changes on immune cells. Here, we took advantage of microarray technology to investigate transcriptomic changes induced by four different $f$-CNTs in human immune cells. THP1, a monocytic cell line, and Jurkat cells, a T lymphocyte cell line, were selected as innate and adaptive immune-cell models, respectively. Data were validated by real-time PCR of selected genes. ELISA of critical soluble factors expressed by monocytes following CNT exposure was also performed on primary isolated human cells.

\section{Material and methods}

\subsection{Carbon nanotubes}

The first type of purified MWCNTs was purchased from NanoAmor (Nanostructured \& Amorphous Materials Inc., Houston, USA). MWCNTs used in this study were $95 \%$ pure (stock No. $1240 \mathrm{XH}$ ). Outer average diameter is between 20 and $30 \mathrm{~nm}$, and length between 0.5 and $2 \mu \mathrm{m}$ before oxidative treatment. These nanotubes were used to synthesize functionalized MWCNTs of type 1 as reported in Ref. [20] (see Fig. S1 for their structures). The second type of nanotubes was obtained from Nanocyl (Sambreville, Belgium) (Thin MWCNT 95\% C purity, Nanocyl 3100 ${ }^{\circledR}$, batch $n^{\circ} 071119$ ). The average diameter and length are $9.5 \mathrm{~nm}$ and $1.5 \mu \mathrm{m}$ respectively. These nanotubes were used to prepare functionalized MWCNTs of type 2 as reported in Ref. [20] (see Fig. S1 for their structures). Characterization of the different functionalized MWCNTs was previously reported [22].

CNTs were homogeneously dispersed at $1 \mathrm{mg} / \mathrm{ml}$ in sterile ultrapure water. Initially, CNTs were sonicated 45 min with a Branson 3200 water bath sonicator and vortexed for a few seconds. An additional sonication was performed for $15 \mathrm{~min}$ before each experiment.

\subsection{Cell cultures}

Jurkat cells (a T cell line), THP1 cells (a monocytic cell line) and isolated human primary monocytes were cultured in RPMI 1640 medium containing $1 \%$ antibioticantimycotic mixture and 10\% heat-inactivated fetal bovine serum (FBS) (Invitrogen).

Human primary cells were obtained from informed healthy male donors (25-50 years old). Cell separation and experiments were performed immediately after blood drawing. Peripheral blood mononuclear cells (PBMCs) were isolated from fresh heparinized blood by Ficoll-Paque PLUS (GE Healthcare) density gradient centrifugation. The experiments were performed on cells from at least 3 different donors. Monocytes were isolated from PBMCs to assess cytokine secretion. Briefly, PBMCs $\left(5 \times 10^{5}\right.$ cells) were incubated in a 48 well plate in RPMI. After $24 \mathrm{~h}$ monocytes attached to the bottom of the wells were washed 6 times with RPMI without FBS. Cells were then incubated in RPMI with the different MWCNTs (100 $\mu \mathrm{g}$ $\mathrm{ml}$ ) or left untreated; supernatants were collected after $24 \mathrm{~h}$. For multiple cytokines secretion assay THP1 cells were centrifuged at $1200 \mathrm{rpm}$ for $10 \mathrm{~min}$.

\subsection{MWCNT uptake assay}

Cells were incubated in a 48 well plate $\left(7 \times 10^{5}\right.$ cells/well $)$ with increasing amounts of fluorescently labeled MWCNTs (MWCNT-FITC 1 and 2) $(1,10,100 \mu \mathrm{g} / \mathrm{ml}$ ) for $24 \mathrm{~h}$. Cells were then washed in PBS. Flow cytometry measurements were performed using a FACSCalibur ${ }^{\circledR}$ and analyzed with CELLQuest software (BD Biosciences). Cell fluorescence was measured after washing with $0.4 \%(\mathrm{w} / \mathrm{v})$ trypan blue to quench fluorescein signal from non internalized CNTs as previously reported [25]

\subsection{Apoptosis and necrosis assay}

To detect cells undergoing apoptosis and necrosis, Annexin-V FITC and propidium iodide staining were employed. The assay is based on the binding of Annexin V to phosphatidylserines (PS) found on the extracellular side of membrane in early apoptotic cells and propidium iodide ability to cross the cell membrane of dead cells Cells were incubated for $24 \mathrm{~h}$ with MWCNTs $(100 \mu \mathrm{g} / \mathrm{ml})$ or left untreated. Jurkat and THP1 cells were then collected and washed with PBS pH 7.2. Staining was performed for $20 \mathrm{~min}$ in the dark. After washing, cells were analyzed by flow cytometry.

\subsection{RNA analysis and microarrays}

Total RNA was extracted using the TriZol reagent (TriZol, Invitrogen, Carlsbad, CA, USA) and further purified using the RNAeasy mini kit following the RNA cleanup protocol as indicated by the manufacturer (Qiagen, Valencia, CA, USA). RNA purity was assessed by spectrophotometric analysis and integrity by microfluidic molecular sizing using the Bioanalyzer 2100 (Agilent). Samples with RIN (RNA Integrity Number $)<8$ were discarded and not used in the gene expression analysis. One microgram of total RNA was converted in cRNA and labeled as described in the Affimetrix GeneChip ${ }^{\circledR}$ Whole Transcript (WT) Sense Target Labeling Assay Manual. We made use of the Affymetrix technology to analyze the expression of 32,020 RefSeq coding transcript with well-established annotations, using the Human Gene 1.0 ST Array (Affymetrix, Santa Clara, CA, USA) following standard protocols. Hybridised Genechips were processed as previously described $[26,27]$. Experiments were performed in triplicate.

\subsection{Real-time $P C R$}

Real-time PCR was performed as follows: $1 \mu \mathrm{g}$ of total RNA was reverse transcribed using the superscript II reverse transcription kit (Invitrogen) following standard protocols. The cDNA reaction $(20 \mu \mathrm{l})$ was brought to a $100 \mu \mathrm{l}$ volume with $\mathrm{H}_{2} \mathrm{O}$ and $1 \mu \mathrm{l}$ used for each PCR reaction. Real-time PCR was performed on an Applied Biosystems 7300 thermal cycler, using the Applied Biosystems real-time PCR master mix without UNG and the following premade Taqman gene expression assays: GAPDH Hs99999905_m1 and ACTB Hs99999903_m1 as housekeeping genes, TNFAIP3 Hs00234713_m1, TNFAIP6 Hs01113602_m1, ICAM1 Hs00164932_m1, NFkB1 Hs00765730_m1, CCL4 Hs01031494_m1, CXCL11 Hs00171138_m1, IL1B Hs01555410_m1. All experiments were performed in triplicate. Gene expression measurements were computed by the $\Delta \mathrm{cT}$ method [28].

\subsection{Multiplex cytokine analysis}

Cell culture supernatants from THP1 cells and isolated human primary monocytes were used to quantify the production of cytokines using a MILLIPLEX MAP 5plex Cytokine Kit (HCYTOMAG-60K -05, Millipore, Billerica, MA), according to manufacturer's protocol. The following human cytokines were measured: IL1 $\beta$, TNF $\alpha$, IL6, and IL10. Briefly, supernatants were centrifuged for $10 \mathrm{~min}$ to remove debris and $25 \mu \mathrm{l}$ were added to $25 \mu \mathrm{l}$ of assay buffer. Then, $25 \mu \mathrm{l}$ of magnetic beads coated with specific antibodies were added to this solution and incubated for $2 \mathrm{~h}$ under shaking. At the end of the incubation, the plate was washed twice in buffe 
and incubated for $1 \mathrm{~h}$ with $25 \mu \mathrm{l}$ of a secondary biotinylated antibody at room temperature. Then, the plate was incubated for 30 min with StreptavidinPhycoerythrin, washed twice, and incubated with $150 \mu \mathrm{l}$ of sheath fluid for $5 \mathrm{~min}$. The plate was observed immediately on a Luminex ${ }^{\circledR} 100^{\mathrm{TM}} / 200^{\mathrm{TM}}$ platform (Luminex Corporation) with xPONENT 3.1 software. Standard curves for each cytokine (in duplicate) were generated by using the supplied reference cytokine concentrations. Cytokine/chemokine concentrations in the samples were determined with a 5parameter logistic curve. Final concentrations were calculated from the mean fluorescence intensity and expressed in $\mathrm{pg} / \mathrm{ml}$. The assay was performed in a 96 -well plate, using all the assay components provided in the kit. All incubation steps were performed at room temperature and in the dark.

\subsection{Statistical analysis}

Statistical analyses for real-time and multiple cytokine assay were performed using Student's $t$-test. Data indicated with a star were considered statistically significant (two-side $p$ value $<0.05$ ). Data are presented as mean $\pm \mathrm{SD}(N=3)$. FACS results are representative of one experiment out of at least 3 different incubations for each sample. Multiplex ELISA tests on isolated human primary monocytes were performed in samples from at least 3 different donors. Gene expression measurements were extracted and normalized from CEL files using the RMA algorithm implemented in Affymetrix Expression Console. The same software was used for QC analysis of the genechips. Statistical analysis and visualization of gene expression data were performed using BRBArrayTools, developed by R. Simon and the BRBArrayTools Development Team [29]. To define the gene expression changes induced by the different CNTs we computed the probability of genes being differentially expressed between the classes using the random variance $t$ test as implemented in BRBArrayTools. Genes were considered statistically significant if $p<0.001$. Per gene false discovery rate was computed using the method of Benjamini and Hochberg [30]. Significant genes were clustered and displayed as heat map using the clustering tool in BRB ArrayTools. These genes were uploaded to Ingenuity Pathway Analysis and analyzed for over-representation of functional categories. Gene expression data was overlaid to significant canonical pathways and visualized as network graph (see Fig. S2). Moreover data interpretation was carried out using the key online resources and tools in biomarkers research [31].

\section{Results}

In order to investigate the genome modulation on innate and adaptive immune cells by functionalized CNTs we used a series of
MWCNTs differing in terms of both functional groups and diameter (Fig. S1). MWCNTs of type 1 are thicker with a diameter between 20 and $30 \mathrm{~nm}$ and an average length of $403 \mathrm{~nm}$, while MWCNTs of type 2 have a smaller diameter $(9.5 \mathrm{~nm})$ and an average length of $396 \mathrm{~nm}$ [22]. Nanotubes were initially oxidized to obtain ox-MWCNT-1 and ox-MWCNT-2 and further modified by 1,3-dipolar cycloaddition reaction to ammonium-functionalized ox-MWCNT- $\mathrm{NH}_{3}^{+}-1$ and oxMWCNT-NH $H_{3}^{+}-2$. Fluorescently labeled nanotubes (ox-MWCNTFITC-1 and ox-MWCNT-FITC-2) used to assess cell internalization were prepared by coupling the ammonium functions of oxMWCNT-NH $\mathrm{N}_{3}^{+}-1$ and ox-MWCNT-NH ${ }_{3}^{+}-2$ with fluorescein isothiocyanate (Fig. S1) [20].

Because we wanted to observe the possible impact on genome expression of MWCNTs when they are internalized into cells, we first investigated the uptake of MWCNTs by Jurkat T and monocyte THP1 cells (Fig. S3). Both types of MWCNTs were internalized in a dose dependent manner with a plateau at $100 \mu \mathrm{g} / \mathrm{ml}$. The uptake of the smaller diameter ox-MWCNT-FITC-2 appears to be facilitated already at low concentrations. These results led us to choose a working concentration of $100 \mu \mathrm{g} / \mathrm{ml}$ CNTs for the following experiments. Twenty-four hours of incubation was fixed as the best time point to see a possible modulation of genes correlated with immune responses.

Because we wanted to perform the genome-wide study in ideal viability conditions, we first performed a necrosis and apoptosis assay (Fig. S4). All MWCNTs tested were proved to be non toxic. Therefore, incubations for the microarray analyses were conducted at $100 \mu \mathrm{g} / \mathrm{ml}$ concentration. Treating immune cells with carbon nanotubes did not induce profound gene expression alteration, at the concentration used. However, a number of transcripts changed in their expression levels following MWCNT treatment (Fig. 1a). We reported a significant change in 74 transcripts in T cells and in 328 in THP1 monocytes. In both types of cells the major impact was due to the treatment with ox-MWCNT-2 with 47 and 212 altered

a

CNT

\begin{tabular}{|c|c|c|c|}
\hline \multirow{2}{*}{$\begin{array}{l}\text { diameters } \\
20-30 \mathrm{~nm}\end{array}$} & & T cells & Monocytes \\
\hline & ox-MWCNT-1 & 25 & 44 \\
\hline & ox-MWCNT-2 & 47 & 212 \\
\hline & ox-MWCNT- $\mathrm{NH}_{3}{ }^{+}-1$ & 1 & 53 \\
\hline $9.5 \mathrm{~nm}$ & ox-MWCNT-NH ${ }_{3}{ }^{+}-2$ & 1 & 19 \\
\hline
\end{tabular}

b

T cells

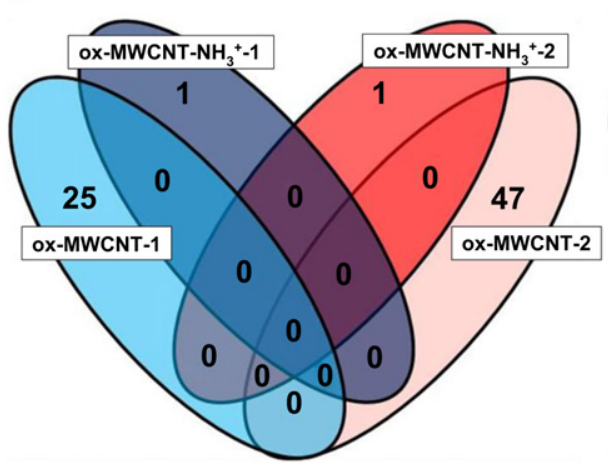

Monocytes

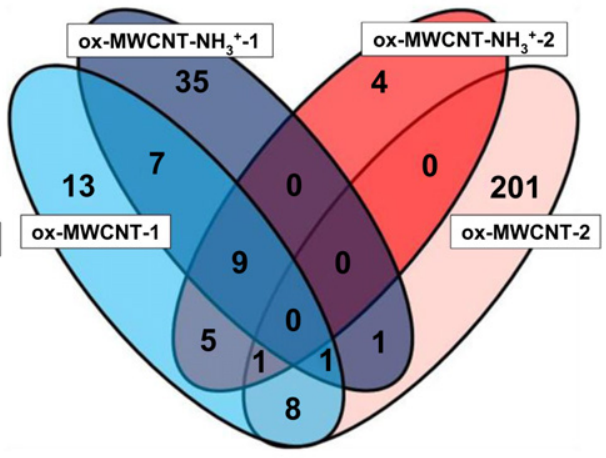

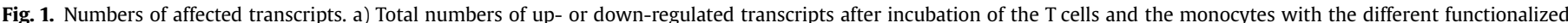

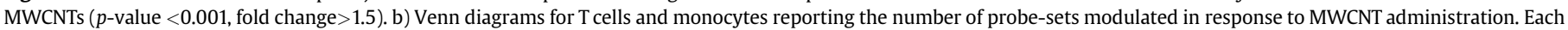
Venn diagram is divided in four areas, one for each type of MWCNTs. Overlapping areas indicate the number of transcripts commonly changed in their expression level. 
transcripts in Jurkat and THP1 cells, respectively. We compared changes in transcript expression induced by the four types of MWCNTs focusing on the common changes (Fig. 1b). Our data indicate that in T cells the effect of ox-MWCNT-2 on gene expression is distinct from that of the other CNTs, as shown by the Venn diagram (Fig. 1b). In monocytes we observed instead different overlapped areas. Table S1 shows the number of transcripts that were commonly changed upon the treatment with different types of MWCNTs. No transcripts were affected by all four CNTs. However, ox-MWCNT-1, ox-MWCNT- $\mathrm{NH}_{3}^{+}-1$ and ox-MWCNT-NH $\mathrm{N}_{3}^{+}-2$, are the most cohesive group with 9 commonly changed transcripts. In Table S2 we reported the list of genes that were affected after treatment with the different MWCNTs in T cells and monocytes. The main effect of ox-MWCNT-2 on both cell types was the modulation of genes encoding ribosomal proteins. The scant overlap observed between CNTs at a single gene level was at least in part due to the stringency of statistical analysis. In order to have a more reliable overview of the effect of each CNT in respect to the others, we generated gene heat maps including all the genes significantly modulated by each CNT in comparison to the controls (cells incubated with medium). Fig. 2 displays the results for T cells. The heat map shows the green area of transcripts down-expressed by oxMWCNT-2, where 15 out of 25 affected transcripts (more than half of the total) code for ribosomal proteins. Fig. S5A shows the heat map for total affected transcripts in monocytes and gives an overview on the broad effect of CNTs in immune cells indicating that ox-MWCNT-2 acts in a more distinct way than the other three CNTs but with similar effect on T cells and monocytes. As displayed in Fig. S5 (B and C, violet and blue rectangles) the modulation of genes coding for ribosomal proteins exerted by ox-MWCNT-2 in T cells was mirrored in monocytes. Nine transcripts correlate to the mitochondrial ribosomal protein family and 26 are linked to ribosomal protein family. Part of these transcripts was down-regulated in monocytes also by ox-MWCNT-1 (Fig. S5, blue rectangles). Moreover,for monocytes we analyzed the upper part of Fig. S5A, a cluster of transcripts activated by the three types of MWCNTs (Fig. 3a) highly enriched in immune-related transcripts, namely oxMWCNT-1, ox-MWCNT-NH $\mathrm{H}_{3}^{+}-1$ and ox-MWCNT-NH ${ }_{3}^{+}-2$. We named these CNTs as monocyte activating CNTs (MA-CNTs) since they induce the expression of several immune transcripts, including genes encoding CCR5 (CCL3L1 and CCL4L1) and CXCR3 ligands (CXCL9, CXCL10 and CXCL11). To investigate the related signaling pathways, we performed an ingenuity analysis (Fig. 3b). Interestingly, we found that the transcripts over-expressed by treating cells with MA-CNTs were closely related to inflammation signaling pathways. Histograms show the most statistically relevant signaling pathways, including bacterial and virus recognition, IL6, CD40, dendritic cell maturation, TNF $\alpha /$ TNFR1-2, and NFkB signaling. Fig. $3 c$ reports the IL1 $\beta$ transduction pathway and shows the other cytokines, kinases and transporters related to NKFB network. To better examine the common gene modulation of MACNTs, we analyzed them in concert (Fig. 4a), by averaging gene fold change induced by each CNT. The top three affected genes were CCL4L1, IL1 and tumor necrosis factor alpha-induced protein 6 (TNFAIP6), with a fold change of 4.07, 3.64 and 3.44, respectively. To validate microarray data, we performed real-time PCR with highly specific Taqman probes for selected genes. We first focused on the top three genes mentioned above (Fig. 4b). We found again these genes consistently over-expressed in MA-CNT samples only. To further confirm the microarray data we assessed real-time PCR for CXCL10, CXCL11, NFkB1A, ICAM1 and TNFAIP3, confirming the genome-wide results (data not shown). CXCL11 was found to be induced in MA-CNT samples with a fold change of 1.74 and 1.54 for the ammonium-functionalized CNTs. We have reported in Fig. S2 the CXCL11 network pathway. In red are highlighted the over-

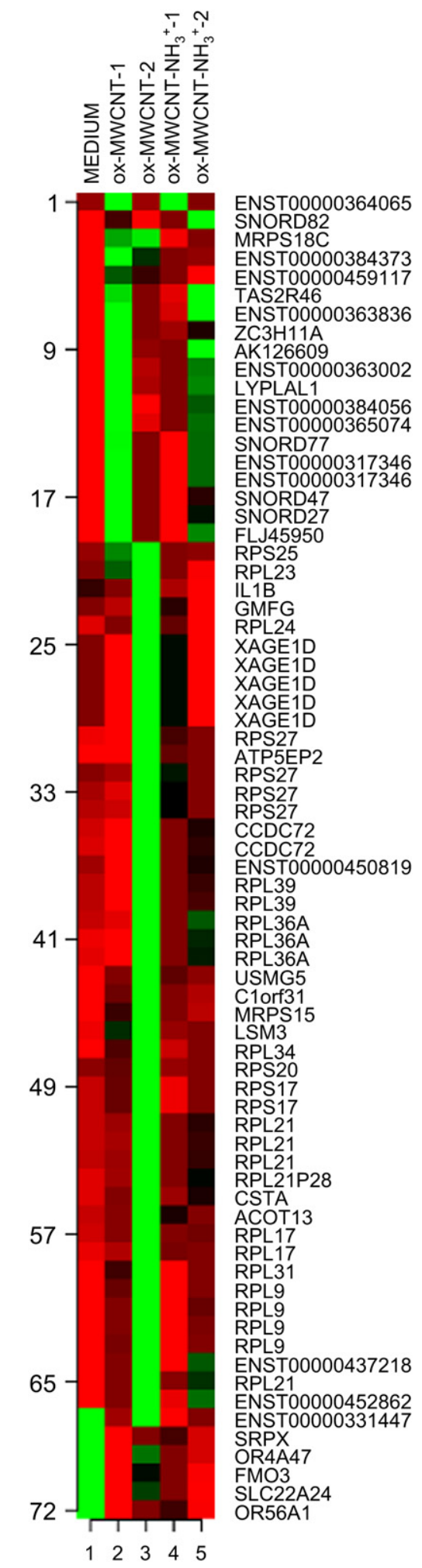

Fig. 2. Gene expression analysis. Heat map of the 74 transcripts modulated in T cells. Genes differentially expressed under the different conditions were clustered and displayed as heat map where individual elements of the plot are colored by their standardized expression values (red $=$ high expression, green $=$ low expression). Geneexpression values used for the heat map were obtained as the mean of triplicate experiments. Heat map was obtained by merging the three transcript lists of each sample. 
a

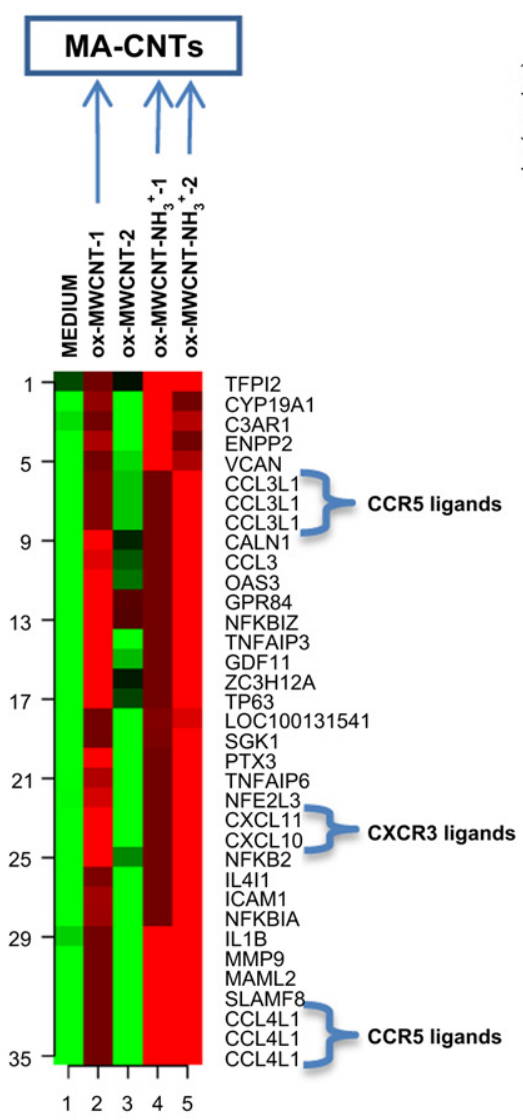

Cytokine

Enzyme

Kinase

Transcription Regulator

Translation Regulator

Transmembrane Receptor

Transporter

Complex / Group

microRNA

\section{Other}

b - Ratio
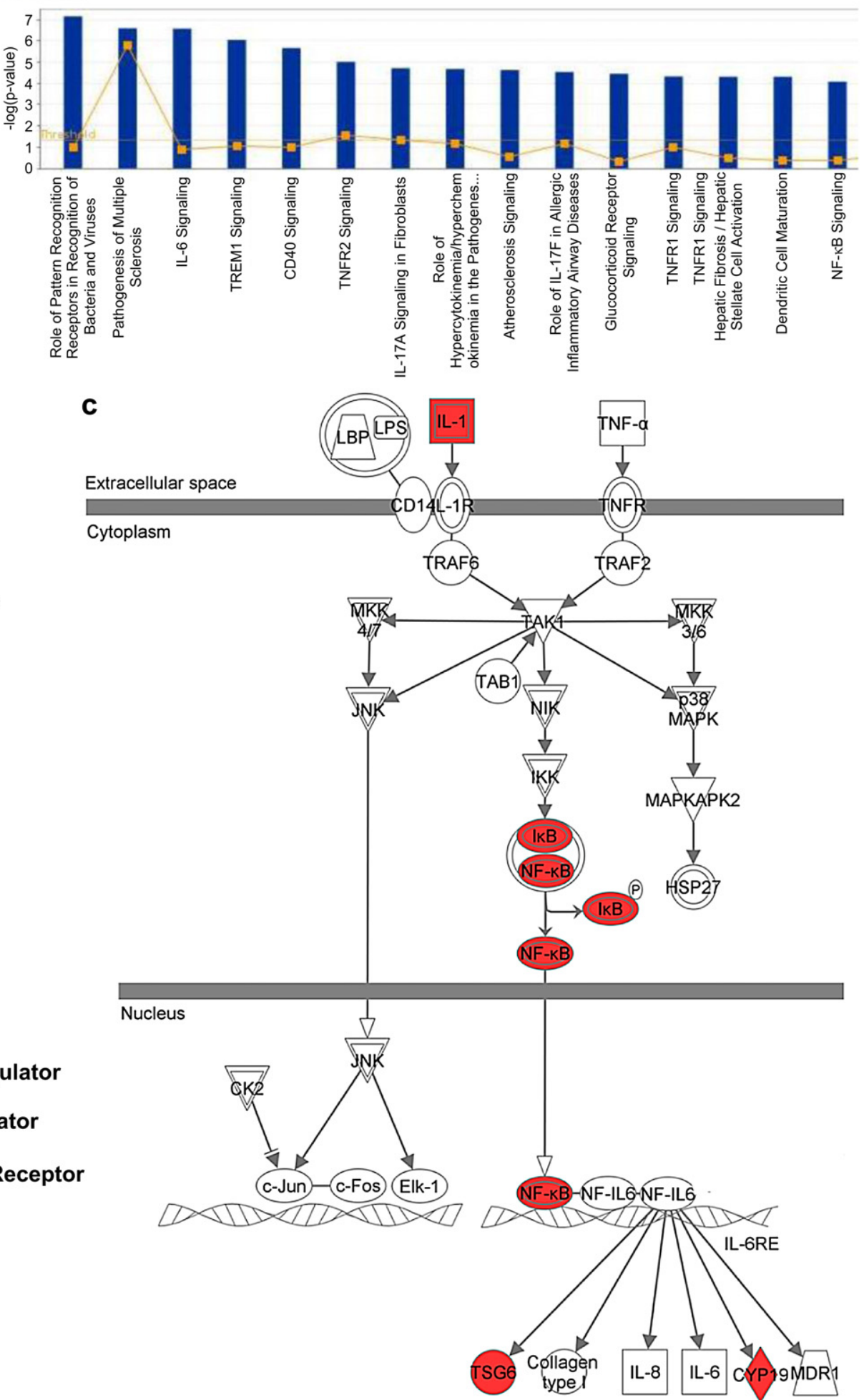

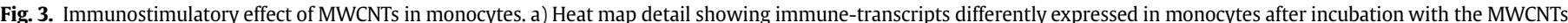

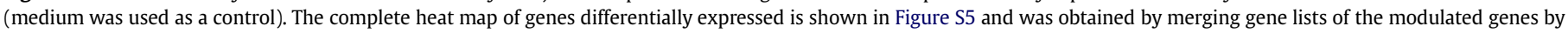

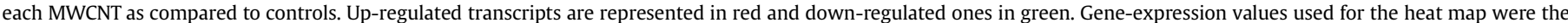

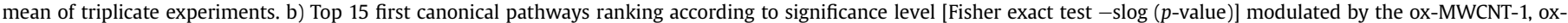

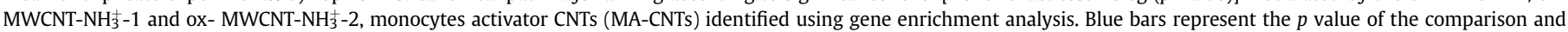

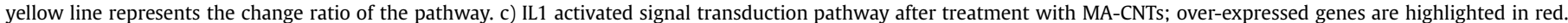




\begin{tabular}{|l|l|c|c|}
\hline GENE & \multicolumn{1}{|c|}{ Function } & p-value & $\begin{array}{c}\text { fold } \\
\text { change }\end{array}$ \\
\hline CCL4L1 & $\begin{array}{l}\text { Chemokine (C-C motif) } \\
\text { ligand 4-like 1 }\end{array}$ & 0.0006926 & 4.07 \\
\hline \hline IL1B & Interleukin 1, beta & 0.0000424 & 3.64 \\
\hline \hline TNFAIP6 & $\begin{array}{l}\text { Tumor necrosis factor, } \\
\text { alpha-induced protein 6 }\end{array}$ & 0.0008174 & 3.44 \\
\hline
\end{tabular}

b
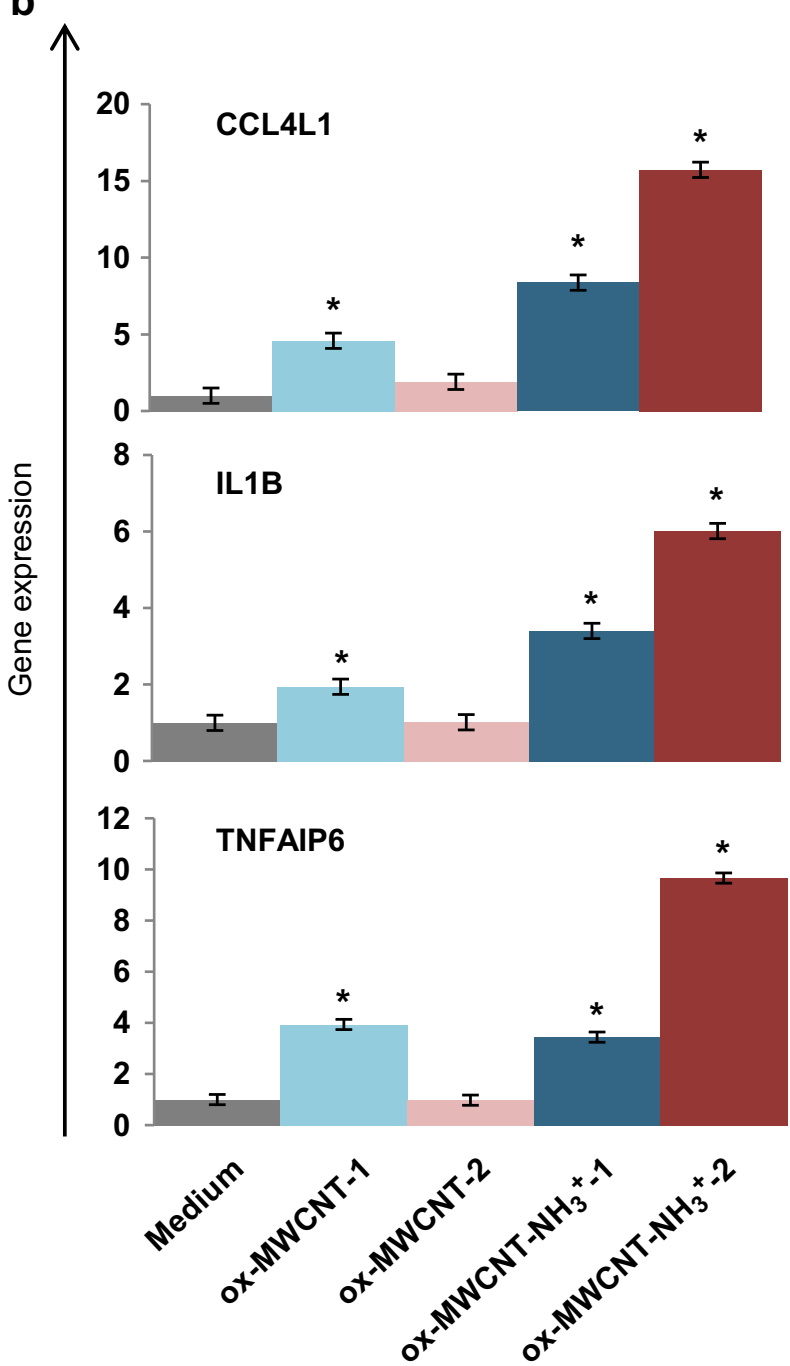

Fig. 4. Genes related to the activation of monocytes. a) Genes commonly induced by MA-CNTs analyzed in concert. b) mRNA levels were measured in triplicate by quantitative real-time PCR. Histograms show residual mRNA level expressed as $\triangle \mathrm{ct}$ MWCNT samples/control medium. The statistical significance of differences between the samples was calculated on the raw data by two-tailed paired data Student's $t$ test. ${ }^{*} p<0.05$.

expressed genes and gene groups including CXCL10 and NFkB1A (for symbol legend see Fig. 3c).

We finally decided to more thoroughly investigate, by ELISA multiplex technology, the secretion, of distinctive cytokines associated to monocyte activation (i.e. IL6, IL1 $\beta$, TNF $\alpha$ and IL10) (Fig. 5) in THP1 cells and also in primary monocytes. In accordance to microarray data and to ELISA assay previously shown on primary monocytes [22], IL6 secretion in THP1 cells treated with the MACNTs ox-MWCNT-NH $H_{3}^{+}-1$ and ox-MWCNT- $\mathrm{NH}_{3}^{+}-2$ was statistically
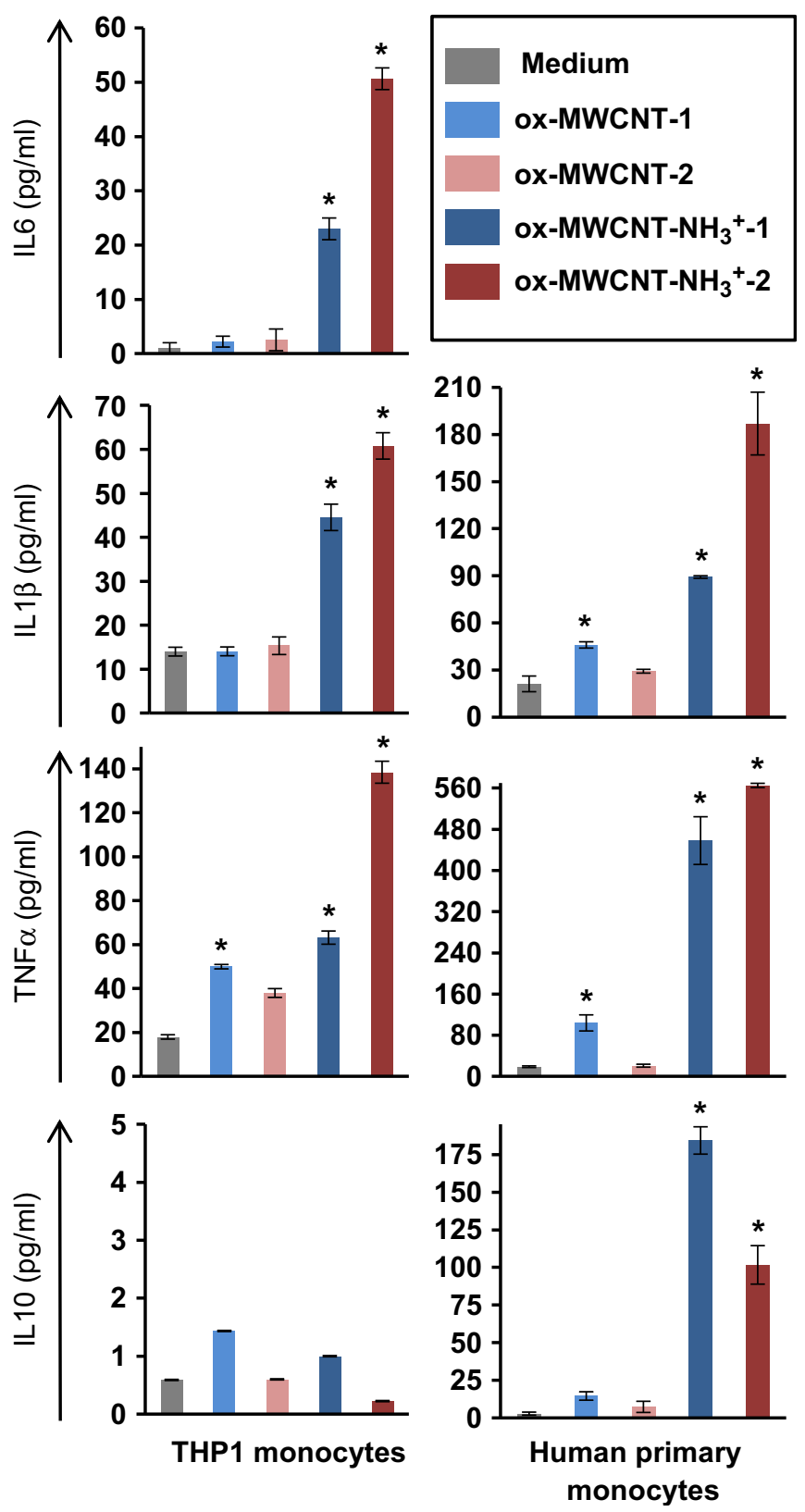

Fig. 5. Cytokine secretion assessed by ELISA multiplex. Cell activation was assessed measuring cytokine production on THP1 monocytes and on isolated human primary monocytes. Values are expressed in $\mathrm{pg} / \mathrm{ml}$. The statistical significance of differences between the samples was calculated by two-tailed paired data Student's $t$-test. ${ }^{*}<0.05$. Data are expressed as mean of triplicate experiments.

increased when compared to controls $(p<0.05)$. Analogous results were found for IL1 $\beta$ secretion and they were confirmed in primary monocytes. TNF $\alpha$ secretion increased in both THP1 cells and primary monocytes after MA-CNT treatment. We further analyzed IL10 secretion. On THP1 cells, no induction of IL10 was observed by any of the CNTs investigated. Interestingly, MA-CNTs enhanced IL10 release in primary monocytes.

\section{Discussion}

In the present work we have performed a genome-wide study on the effect of four types of functionalized MWCNTs on cells representative of the adaptive (Jurkat $\mathrm{T}$ cell) and innate (THP1 monocytes) immune response, after having assessed their uptake 
and excluded the possible cytotoxic effects. We observed that the most striking changes occurred in THP1 cells and involved the expression of functionally relevant immune genes. Our thorough investigation on the molecular effects of MWCNTs establishes that they do not induce any toxicogenomic effect. Surprisingly, we found that the immune interaction of ox-MWCNT- $\mathrm{NH}_{3}^{+}-1$, oxMWCNT-NH ${ }_{3}^{+}-2$ and ox-MWCNT-1 (MA-CNTs) is cell and pathway specific, with a direct effect on monocyte activation, while a negligible effect was observed in $\mathrm{T}$ cell activation. The gene expression profiles in monocytes treated with MA-CNTs was related to a boost of the early innate response resulting in a fingerprint characterized by the enhancement of pathways that are classically activated during response to infection and vaccination, allograft rejection and anti-tumor immune response (i.e. dendritic cell maturation, IL1 $\beta$, IL6, CD40, NFkB, TNF $\alpha$, and CXCR3/ CCR5-ligand signaling pathways) (Fig. 3) [32].Conversely, oxMWCNT-2, which have a small diameter $(9.5 \mathrm{~nm})$ and lack triethylene glycol chain modulates ribosomal genes both in monocytes and $\mathrm{T}$ cells.

We validated the microarray data by Taqman real-time PCR for selected transcripts. For this purpose, we carried out multiplex ELISA in THP1 cells and human primary monocytes. We confirmed IL6 results previously reported for primary monocytes [22]. Overall, MA-CNTs induced secretion of IL1 $\beta$ and TNF $\alpha$ in both THP1 and primary monocytes. Although IL6 and TNF $\alpha$ downstream effects were shown by the gene-expression pathway analysis, their individual mRNA expression levels (assessed $24 \mathrm{~h}$ after incubation) were not significantly overexpressed. This is clearly due to the kinetic of expression of IL6 and TNF $\alpha$ mRNA, whose peak under stimulus is reached at 4-6 h [33]. Interestingly, we also found an increased IL10 release in primary cells treated with ox-MWCNT$\mathrm{NH}_{3}^{+}-1$ and ox-MWCNT- $\mathrm{NH}_{3}^{+}-2$, not observed in THP1 cells. This discrepancy could be probably due to the high polymorphism of IL10 promoter, with consequent differential IL10 modulation [34].

Several studies were performed to date to understand the possible effect on the immune system of pristine or functionalized CNTs. However, most of them focused on the CNT impact on the traditional immunity markers such as cytokine expression [15-17]. Therefore, following this approach, if we keep in mind that the current number of protein-codifying human genes is about 20,000 [35], it can be hard to have a comprehensive overview of the molecules modulated by CNT treatment. To our knowledge only few studies, related to CNTs, used whole-genome gene expression profiling. All of them assessed the impact of non-functionalized nanotubes, and reported strong cytotoxic effects. Alazzam et al. compared the profile of human normal bronchial epithelial cells with that of cells treated with SWCNTs [36]. The large amount of genes modulated by single-walled CNTs (about 14.000) is likely due to the absence of correction for multiplicity and to selection of a relaxed $p$ value (the cut-off for significance was not specified in that study). With this limitation, most of the genes that the authors found over-expressed in single-walled CNT-treated cells were related to cell apoptosis, while genes involved in cell proliferation and survival were down-regulated, implying a strong association with cytotoxicity. Ding et al. focused on human skin fibroblasts [37]. Their objective was a comparison between the effects of carbon nano-onions and non-functionalized MWCNTs. Microarray analysis after stringent correction for multiplicity showed perturbation of multiple cellular pathways involving 46 modulated genes. Considering that the concentration utilized by Ding et al. was more than 100 times lower than that used in the present study, the total number of genes changed in their expression is considerably elevated compared to that detected in our analysis. In fact, the major impact of the treatment in terms of number of genes was reached by the ox-MWCNT-2 and resulted in the modulation of 212 transcripts in monocytes and 47 in $\mathrm{T}$ cells, followed by the oxMWCNT- $\mathrm{NH}_{3}^{+}-1$, which modulated 53 genes in monocytes and only one gene in T cells. It should be emphasized that we interrogated more than 30.000 transcripts covering the whole genome, and that only few of them resulted altered by MWCNTs. Similarly to our results, the same authors detected the expression of several IFN stimulating genes. Conversely to our findings, this immune activation was accompanied by the induction of cycle arrest and apoptotic processes. To our knowledge, only Chou et al. performed a genome-wide analysis on immune cells [24]. The treatment of THP1-derived macrophages with non-functionalized single-walled CNTs showed activation of various transcription factors such as NFkB and activator protein 1 (AP-1) and the release of proinflammatory and chemoattractant cytokines. Several other pathways induced by the non-functionalized SWCNTs used by Chou et al. overlapped with those induced, in the present study, by the MA-CNTs, including TNF $\alpha$, IL1 $\beta$, CD40 and CXCR3/CCR5 ligand pathways. However, that activation was accompanied by considerable toxicity underlined by the activation of pathways associated with the oxidative stress and the caspase cascade. It is not surprising that such CNTs, in view of the lack of water solubility and because of their structure, induce substantial cytotoxicity. The authors ascribed the observed in vitro activation of NFkB as a countermechanism to neutralize the oxidative stress and the pro-apoptotic stimulus found by the pathway analysis. Conversely, when functionalized CNTs were employed, we observed the activation of NFkB in the absence of oxidative stress and pro-apoptotic induced pathways, providing evidence that the immune activation of MACNTs is an intrinsic characteristic of these materials and not secondary to the induction of pro-apoptotic/cytotoxic events. Therefore, functionalized MA-CNTs exquisitely act as immune activators of monocytes.

Interestingly, MA-CNTs mimic several effects induced by IFN- $\gamma$, the main monocyte-macrophage activator molecule. In fact several genes expressed by monocytes following MA-CNT treatment (i.e. IL1B, IL-6, MMP9, ICAM1, CXCL9, CXCL10 and CXCL11) can be induced, directly or indirectly, by IFN- $\gamma$ stimulus [38-40]. The interaction between TNF $\alpha$, NFkB, IL1, IL6, and CXCR3/CCR5 pathway is complicated, in view of the existence of several autocrine loops shared by these pathways. NFkB activity is stimulated by proinflammatory cytokines, such as TNF $\alpha$ and IL1, as well as by pathogen-associated molecular patterns (PAMPs, present in viral and microbial strains). NFkB can, in turn, induce the expression of IL6, and of CXCR3 andCCR5 ligands. IL1 can also induce TNF $\alpha$, which can in turn induce IL6 [41-43]. Although it is not clear which is the first target engaged by MA-CNTs, their downstream effect mimic that triggered by pathogen strains through Toll-like receptors.

The study of the ability of MA-CNTs to powerfully activate CXCR3/CCR5 ligand pathways (i.e. CXCL9, CXCL10, CXCL11, CCL3L1 and CCL4L1, which encode CCL3 and CCL4 isoforms, respectively) deserves particular attention, in view of the functional relevance of these pathways. Indeed, the recruitment of immune cells represents a necessary condition for the development of an effective anti-tumor response. Several genomic and proteomic studies showed that the main mechanism of action of immunotherapeutic agents (i.e. anti-CTLA4 mAbs or IL2) used for cancer therapy is represented by the induction of an acute inflammation at the tumor site, with consequent activation of monocyte function and release of high quantities of specific Th1 chemokines (CXCR3 and CCR5 ligands). These chemokines, by binding the corresponding receptor, recruit activated CD8 T cells, T helper 1 cells and NK cells which can mediate tumor rejection [32,44-46].

As future perspective, the ability of MA-CNTs to elicit these pathways could be investigated in preclinical studies. Experimentally, MA-CNTs might be administered systematically and their 
localization at the tumor level could be confirmed by ultrasonography in view of their echogenic characteristic, as recently described by our group [14].

We used four types of multi-walled CNTs to take into consideration and explore the effects of some important parameters of CNTs and nanomaterials in general, namely size and surface properties. The impact of length on biocompatibility of the nanotubes is being well studied $[1,47,48]$. The effect of diameter on biocompatibility, especially regarding the possible role in the impact on the immune system, has not been adequately determined yet, likely because length was a more attractive issue due to the analogy between long carbon nanotubes and asbestos fibers [49]. Sohaebuddin et al. noticed that MWCNTs $>50 \mathrm{~nm}$ are more toxic to RAW macrophages than small diameter MWCNTs ( $<8$ and 20-30 nm) [50]. In contrast to that work, in a more recent study thin MWCNTs $(9.4 \mathrm{~nm}$ ) resulted significantly more toxic than the thicker tubes $(70 \mathrm{~nm})$ both in vitro in macrophages and in vivo in a mouse model [51]. However, in contrast to our study, where lengths were clearly defined (about $400 \mathrm{~nm}$ for both type 1 and type 2 MWCNTs), the authors reported a large length range $(0.1-3 \mu \mathrm{m})$, which clearly represents a bias in the interpretation of the results. Oppositely, it has been previously reported that MWNTs of small diameter showed less cytotoxicity than large nanotubes in guinea pig alveolar macrophages [52]. With the present study we confirmed, expanded, and explained our previous findings [22]. When MWCNTs are oxidized and further functionalized with ammonium groups, independently of the difference in diameter, they boost the innate immune response. We observed that the combination of two characteristics: 1) the low diameter ( $9.5 \mathrm{~nm})$, and 2) the absence of ammonium groups, determines the lack of immunostimulatory properties as observed for ox-MWCNT-2. The combination of these two factors seems to dramatically change the biological activity. Dispersibility could be an additional factor as ox-MWCNT-2 disperse better in water compared to ox-MWCNT-NH $\mathrm{H}_{3}^{+} 2$. We believe that the action of CNTs on the immune system is not a black or white picture but it is instead a variegated image where several factors, including functionalities and diameter, act in concert to determine their biological activity.

Generally, nanoparticles and/or microparticles are promising vaccine delivery systems with potential adjuvant activity. A vaccine adjuvant is a component that potentiates the immune responses to an antigen and/or modulates it towards the desired immune responses. The potential role of nano/microparticles as adjuvant components has been thoroughly reviewed by Oyewumi et al. [53]. The authors pointed out how much difficult it is to achieve an accurate prediction of particle size ranges that will dictate a Th1 or a mixed Th1/Th2 immune response outcome. However, likely because of the absence of studies in this direction, they did not take into consideration CNTs. Aluminum compounds, especially aluminum phosphate and aluminum hydroxide, are commonly used as vaccine adjuvants [54]. Several of the pathways induced by aluminum hydroxide, overlap with those activated by MA-CNTs and include IL6, IL1 $\beta$, and TNF $\alpha$ pathways. Although aluminum has a long track record of safety, it only improves the induction of a humoral immune response (through a Th2 pathway), and does not help cell-mediated immune responses (Th1 pathway). In contrast, MA-CNTs could enhance a Th1 cellular response through the activation of the specific Th1 chemokine pathways (i.e. CXCR3/CCR5 ligand-induced pathways).

\section{Conclusions}

We investigated the interaction of functionalized CNTs with the immune system, considering a wide variety of parameters, including the whole-genome expression, the two different adaptive and innate responses, and four types of CNTs, analyzed by comparing the same diameters and the different functionalization and/or the different diameters and the same functionalities. None of them induced cytotoxicity and the overall effect on cell metabolism was negligible. We found a cell specific action on monocytes for three types of CNTs, which specifically enhanced innate immunity activation mechanisms. The pathways activated by these nanotubes are functionally relevant and critical for the development of an effective inflammatory response, as that necessary for pathogen clearance and tumor rejection. Despite the common rule that a nanocarrier is good only if inert, we believe that we should not miss the opportunity to test carbon nanotubes as immunomodulator system in different conditions. In this context, our study builds the rationale to test them in preclinical models as immunotherapeutic agents or as adjuvant in vaccine therapy for cancer and infectious diseases.

\section{Acknowledgments}

We thank Alessia Lamolinara (Unità operativa di immunooncologia, Università degli Studi di Chieti) for technical assistance. We thank the laboratories of Prof. Pippia and Prof. Fiori (UNISS) for the kind gift of Jurkat and THP1 cell lines. L.G.D. was supported by "Gianfranco Del Prete award 2012 for Medicine, Biology and Nanotechnology". D.B. was supported by the Conquer Cancer Foundation of the American Society of Clinical Oncology 2011 Young Investigator Award. This work was supported in part by Fondazione Banco di Sardegna Grants 994/2010.0410 and 186/ 2011.0484 (to L.G.D.), the University of Sassari, the Centre National de la Recherche Scientifique, and the European Union Seventh Framework Programme FP7 (HEALTH-2007-201587, ANTICARB) program. L.G.D. wishes to thank Sardinia Region for supporting an Invited Professorship to A.B.

\section{Appendix A. Supplementary data}

Supplementary data related to this article can be found at http:// dx.doi.org/10.1016/j.biomaterials.2013.02.052.

\section{References}

[1] Ali-Boucetta H, Sainz R, Herrero MA, Tian B, Prato M, Bianco A, et al. Asbestoslike pathogenicity of long carbon nanotubes alleviated by chemical functionalization. Angew Chem Int Ed 2013;52:2274-8.

[2] Meng L, Zhang X, Lu Q, Fei Z, Dyson PJ. Single walled carbon nanotubes as drug delivery vehicles: targeting doxorubicin to tumors. Biomaterials 2012;33: 1689-98.

[3] Bonner JC. Carbon nanotubes as delivery systems for respiratory disease: do the dangers outweigh the potential benefits? Expert Rev Respir Med 2011;5: 779-87.

[4] Villa CH, Dao T, Ahearn I, Fehrenbacher N, Casey E, Rey DA, et al. Single-walled carbon nanotubes deliver peptide antigen into dendritic cells and enhance IgC responses to tumor-associated antigens. ACS Nano 2011;5:5300-11.

[5] Herrero MA, Toma FM, Al-Jamal KT, Kostarelos K, Bianco A, Da Ros T, et al Synthesis and characterization of a carbon nanotube-dendron series for efficient siRNA delivery. J Am Chem Soc 2009;131:9843-8.

[6] Pantarotto D, Singh R, McCarthy D, Erhardt M, Briand JP, Prato M, et al. Functionalized carbon nanotubes for plasmid DNA gene delivery. Angew Chem Int Ed Engl 2004:43:5242-6.

[7] Fabbro C, Ali-Boucetta H, Da Ros T, Kostarelos K, Bianco A, Prato M. Targeting carbon nanotubes against cancer. Chem Commun (Camb) 2012;48:3911-26.

[8] Bianco A, Kostarelos K, Prato M. Making carbon nanotubes biocompatible and biodegradable. Chem Commun (Camb) 2011;47:10182-8.

[9] Ménard-Moyon C, Kostarelos K, Prato M, Bianco A. Functionalized carbon nanotubes for probing and modulating molecular functions. Chem Bio 2010;17:107-15.

[10] Delogu LG, Magrini A, Bergamaschi A, Rosato N, Dawson MI, Bottini N, et al Conjugation of antisense oligonucleotides to PEGylated carbon nanotubes enables efficient knockdown of PTPN22 in T lymphocytes. Bioconjug Chem 2009;20:427-31.

[11] Fiorillo E, Orru V, Stanford SM, Liu Y, Salek M, Rapini N, et al. Autoimmune-associated PTPN22 R620W variation reduces phosphorylation of 
lymphoid phosphatase on an inhibitory tyrosine residue. J Biol Chem 2010;285:26506-18.

[12] Burke A, Ding X, Singh R, Kraft RA, Levi-Polyachenko N, Rylander MN, et al. Long-term survival following a single treatment of kidney tumors with multiwalled carbon nanotubes and near-infrared radiation. Proc Natl Acad Sci U S A 2009;106:12897-902.

[13] Cellot G, Cilia E, Cipollone S, Rancic V, Sucapane A, Giordani S, et al. Carbon nanotubes might improve neuronal performance by favouring electrical shortcuts. Nat Nanotechnol 2009;4:126-33.

[14] Delogu LG, Vidili G, Venturelli E, Ménard-Moyon C, Zoroddu MA, Pilo G, et al. Functionalized multiwalled carbon nanotubes as ultrasound contrast agents. Proc Natl Acad Sci U S A 2012;109:16612-7.

[15] Yu Y, Zhang Q, Mu Q, Zhang B, Yan B. Exploring the immunotoxicity of carbon nanotubes. Nanoscale Res Lett 2008;3:271-7.

[16] Boraschi D, Costantino L, Italiani P. Interaction of nanoparticles with immunocompetent cells: nanosafety considerations. Nanomedicine (Lond) 2012;7: 121-31.

[17] Jang J, Lim DH, Choi IH. The impact of nanomaterials in immune system. Immune Netw 2010;10:85-91.

[18] Boncel S, Muller KH, Skepper JN, Walczak KZ, Koziol KK. Tunable chemistry and morphology of multi-wall carbon nanotubes as a route to non-toxic, theranostic systems. Biomaterials 2011;32:7677-86.

[19] Tahara Y, Nakamura M, Yang M, Zhang M, Iijima S, Yudasaka M. Lysosomal membrane destabilization induced by high accumulation of single-walled carbon nanohorns in murine macrophage RAW 264.7. Biomaterials 2012;33:2762-9.

[20] Cheng C, Muller KH, Koziol KK, Skepper JN, Midgley PA, Welland ME, et al Toxicity and imaging of multi-walled carbon nanotubes in human macrophage cells. Biomaterials 2009;30:4152-60.

[21] Dumortier H, Lacotte S, Pastorin G, Marega R, Wu W, Bonifazi D, et al. Functionalized carbon nanotubes are non-cytotoxic and preserve the functionality of primary immune cells. Nano Lett 2006;6:1522-8.

[22] Delogu LG, Venturelli E, Manetti R, Pinna GA, Carru C, Madeddu R, et al. Ex vivo impact of functionalized carbon nanotubes on human immune cells. Nanomedicine (Lond) 2012;7:231-43.

[23] Kaur G, Valarmathi MT, Potts JD, Jabbari E, Sabo-Attwood T, Wang Q. Regulation of osteogenic differentiation of rat bone marrow stromal cells on 2D nanorod substrates. Biomaterials 2010;31:1732-41.

[24] Chou CC, Hsiao HY, Hong QS, Chen CH, Peng YW, Chen HW, et al. Singlewalled carbon nanotubes can induce pulmonary injury in mouse model. Nano Lett 2008;8:437-45.

[25] Delogu LG, Stanford SM, Santelli E, Magrini A, Bergamaschi A, Motamedchaboki $\mathrm{K}$, et al. Carbon nanotube-based nanocarriers: the importance of keeping it clean. J Nanosci Nanotechnol 2010;10:5293-301.

[26] Pescatori M, Broccolini A, Minetti C, Bertini E, Bruno C, D’Amico A, et al. Gene expression profiling in the early phases of DMD: a constant molecular signature characterizes DMD muscle from early postnatal life throughout disease progression. FASEB J 2007:21:1210-26.

[27] Pan Q, Fouraschen SM, Kaya FS, Verstegen MM, Pescatori M, Stubbs AP, et al. Mobilization of hepatic mesenchymal stem cells from human liver grafts. Liver Transpl 2011;17:596-609.

[28] Livak KJ, Schmittgen TD. Analysis of relative gene expression data using realtime quantitative PCR and the 2(-Delta Delta C(T)) Method. Methods 2001;25: 402-8.

[29] Simon R, Lam A, Li MC, Ngan M, Menenzes S, Zhao Y. Analysis of gene expression data using BRB-ArrayTools. Cancer Inform 2007;3:11-7.

[30] Benjamini Y, Drai D, Elmer G, Kafkafi N, Golani I. Controlling the false discovery rate in behavior genetics research. Behav Brain Res 2001:125:279-84.

[31] Bedognetti D, Balwit JM, Wang E, Disis ML, Britten CM, Delogu LG, et al. SITC iSBTc cancer immunotherapy biomarkers resource document: online resources and useful tools - a compass in the land of biomarker discovery. J Transl Med 2011;9:155.

[32] Bedognetti D, Wang E, Sertoli MR, Marincola FM. Gene-expression profiling in vaccine therapy and immunotherapy for cancer. Expert Rev Vaccines 2010;9: 555-65.

[33] DeForge LE, Remick DG. Kinetics of TNF, IL-6, and IL-8 gene expression in LPSstimulated human whole blood. Biochem Biophys Res Commun 1991;174: $18-24$.
[34] Grinyo J, Vanrenterghem Y, Nashan B, Vincenti F, Ekberg H, Lindpaintner K, et al. Association of four DNA polymorphisms with acute rejection after kidney transplantation. Transpl Int 2008;21:879-91.

[35] Genomics Pennisi E. ENCODE project writes eulogy for junk DNA. Science 2012;337(1159):61.

[36] Alazzam A, Mfoumou E, Stiharu I, Kassab A, Darnel A, Yasmeen A, et al Identification of deregulated genes by single wall carbon-nanotubes in human normal bronchial epithelial cells. Nanomedicine 2010;6:563-9.

[37] Ding L, Stilwell J, Zhang T, Elboudwarej O, Jiang H, Selegue JP, et al. Molecular characterization of the cytotoxic mechanism of multiwall carbon nanotubes and nano-onions on human skin fibroblast. Nano Lett 2005;5:2448-64.

[38] Spivey TL, Uccellini L, Ascierto ML, Zoppoli G, De Giorgi V, Delogu LG, et al. Gene expression profiling in acute allograft rejection: challenging the immunologic constant of rejection hypothesis. J Transl Med 2011;9:174.

[39] Harris JE, Fernandez-Vilaseca M, Elkington PT, Horncastle DE, Graeber MB, Friedland JS. IFNgamma synergizes with IL-1beta to up-regulate MMP-9 secretion in a cellular model of central nervous system tuberculosis. FASEB J 2007;21:356-65.

[40] Chang YJ, Holtzman MJ, Chen CC. Interferon-gamma-induced epithelial ICAM1 expression and monocyte adhesion. Involvement of protein kinase Cdependent c-Src tyrosine kinase activation pathway. J Biol Chem 2002;277: $7118-26$.

[41] Sanceau J, Wijdenes J, Revel M, Wietzerbin J. IL-6 and IL-6 receptor modulation by IFN-gamma and tumor necrosis factor-alpha in human monocytic cell line (THP-1). Priming effect of IFN-gamma. J Immunol 1991;147:2630-7.

[42] Ikejima T, Okusawa S, Ghezzi P, van der Meer JW, Dinarello CA. Interleukin-1 induces tumor necrosis factor (TNF) in human peripheral blood mononuclear cells in vitro and a circulating TNF-like activity in rabbits. J Infect Dis 1990;162:215-23.

[43] Bonizzi G, Karin M. The two NF-kappaB activation pathways and their role in innate and adaptive immunity. Trends Immunol 2004;25:280-8.

[44] Weiss GR, Grosh WW, Chianese-Bullock KA, Zhao Y, Liu H, Slingluff Jr CL, et al. Molecular insights on the peripheral and intratumoral effects of systemic high-dose rIL-2 (aldesleukin) administration for the treatment of metastatic melanoma. Clin Cancer Res 2011;17:7440-50.

[45] Carretero R, Wang E, Rodriguez AI, Reinboth J, Ascierto ML, Engle AM, et al. Regression of melanoma metastases after immunotherapy is associated with activation of antigen presentation and interferon-mediated rejection genes. Int J Cancer 2012;131:387-95.

[46] Ji RR, Chasalow SD, Wang L, Hamid O, Schmidt H, Cogswell J, et al. An immune-active tumor microenvironment favors clinical response to ipilimumab. Cancer Immunol Immunother 2012;61:1019-31.

[47] Johnston HJ, Hutchison GR, Christensen FM, Peters S, Hankin S, Aschberger K, et al. A critical review of the biological mechanisms underlying the in vivo and in vitro toxicity of carbon nanotubes: the contribution of physico-chemical characteristics. Nanotoxicology 2010;4:207-46.

[48] Murphy FA, Poland CA, Duffin R, Al-Jamal KT, Ali-Boucetta H, Nunes A, et al. Length-dependent retention of carbon nanotubes in the pleural space of mice initiates sustained inflammation and progressive fibrosis on the parietal pleura. Am J Pathol 2011;178:2587-600.

[49] Donaldson K, Murphy FA, Duffin R, Poland CA. Asbestos, carbon nanotubes and the pleural mesothelium: a review of the hypothesis regarding the role of long fibre retention in the parietal pleura, inflammation and mesothelioma. Part Fibre Toxicol 2010;7:5.

[50] Sohaebuddin SK, Thevenot PT, Baker D, Eaton JW, Tang L. Nanomaterial cytotoxicity is composition, size, and cell type dependent. Part Fibre Toxicol 2010;7:22.

[51] Fenoglio I, Aldieri E, Gazzano E, Cesano F, Colonna M, Scarano D, et al. Thickness of multiwalled carbon nanotubes affects their lung toxicity. Chem Res Toxicol 2012;25:74-82.

[52] Wang X, Jia G, Wang H, Nie H, Yan L, Deng XY, et al. Diameter effects on cytotoxicity of multi-walled carbon nanotubes. J Nanosci Nanotechnol 2009;9:3025-33.

[53] Oyewumi MO, Kumar A, Cui Z. Nano-microparticles as immune adjuvants: correlating particle sizes and the resultant immune responses. Expert Rev Vaccines 2010;9:1095-107.

[54] Marrack P, McKee AS, Munks MW. Towards an understanding of the adjuvant action of aluminium. Nat Rev Immunol 2009;9:287-93. 